\title{
Hospital-Acquired Diabetic Ketoacidosis (HADKA) - An Analysis Comparing Two Major Australian Hospitals
}

\author{
Ushank Ranasinghe ${ }^{1^{*}}$ and Shamasunder Acharya ${ }^{2,3}$ \\ ${ }^{1}$ Endocrine Advance Trainee, John Hunter Hospital, Newcastle, Australia \\ ${ }^{2}$ Director of Diabetes Service, Senior Staff Specialist, John Hunter Hospital, Newcastle, Australia \\ ${ }^{3}$ Conjoint Associate Professor, University of Newcastle, Australia
}

*Corresponding author: Ushank Ranasinghe, Endocrine Advance Trainee, John Hunter Hospital, Newcastle, Australia

\begin{abstract}
Objectives: To identify the factors contributing to hospital-acquired DKA and its adverse outcomes in two Australian hospitals.

Materials and methods: We conducted a retrospective study looking at patients over 18 yrs who developed hospital-acquired Diabetic Ketoacidosis (HADKA) in two major hospitals in Hunter New England Health, NSW Australia between $1^{\text {st }}$ July 2014 and $30^{\text {th }}$ June 2018 . The data were obtained from patient electronic medical records.

The HADKA criteria were $\mathrm{pH} \leq 7.3$, bicarbonate $\leq 18$ $\mathrm{mmol} / \mathrm{L}$, and positive ketones (urine or plasma). A subset of patients was defined as 'inferred HADKA' when there was no documented ketone level, and no other cause of acidosis was identified. We excluded patients who had DKA on admission, and when the acidosis was primarily a result of a different medical condition.
\end{abstract}

Results: There were 12 patients who developed HADKA. The patients were predominantly non-aboriginal $(91.66 \%)$ and elderly. Most had T2DM $(75 \%, n=9)$, and the mean $\mathrm{HbA} 1 \mathrm{c}$ was $8.4 \%$. Majority of the patients developed DKA while admitted under a medical team. The average nadir $\mathrm{pH}$ was 7.19 , bicarbonate was $12.92 \mathrm{mmol} / \mathrm{L}$ and highest glucose was $17.5 \mathrm{mmol} / \mathrm{L}$. An $83.3 \%$ of this population ( $=10$ ) needed ICU management. Time for DKA resolution was 3.33 days. Two (16.6\%) died following the diagnosis of HADKA.

The most common reason for HADKA was related to insulin administration, which included withholding insulin $(n=1$ $8.3 \%)$, insulin dose reduction $(n=3,25 \%)$ and interruption to insulin infusion $(n=1,8.3 \%)$. The remaining cases were caused by infection ( $n=4,33.3 \%$ ), SGLT2 inhibitor use ( $n$ $=3,25 \%)$, steroid-induced hyperglycaemia $(n=1,8.3 \%)$ and acute stress from cardiac disease/cardiogenic shock $(n=2,16.6 \%)$.
Conclusion: Hospital-acquired DKA is a preventable nosocomial complication with high morbidity and mortality. Simple interventions targeting appropriate insulin dosing and early recognition of hyperglycaemia could potentially prevent many cases of HADKA.

\section{Introduction}

Australia has a high prevalence of patients with diabetes who get hospitalised, estimated at over $10 \%$ every year. According to the Australian Institute of Health and Welfare (AlHW) data, there were over 1 million hospitalisations associated with diabetes between 2016 and 2017. Most of them recorded diabetes as an additional diagnosis and in $4 \%$ of patients, diabetes was the principal reason for admission [1].

One of the most feared complications of poorly controlled diabetes is diabetic ketoacidosis (DKA). There were 7,132 hospitalisations diagnosed with DKA [2] in Australia between 2014 and 2015 [2]. Patients with diabetes are at increased risk of serious adverse events, including severe hyperglycaemia during their hospital stay. This includes HADKA, which results from suboptimal management of glycaemia. Many hospitals are using state and local protocols to optimise inpatient glycaemic control. Poor glycaemia in hospitalised patients is associated with increased morbidity and mortality. Despite this notion, hyperglycaemia among hospitalised patients remains prevalent. Observational studies have reported a prevalence of hyperglycaemia ranging from $38 \%$ to $40 \%$ in hospitals and a range of $70 \%$ to $80 \%$ in diabetic patients with critical illnesses

Citation: Ranasinghe U, Acharya S (2021) Hospital-Acquired Diabetic Ketoacidosis (HADKA) - An Analysis Comparing Two Major Australian Hospitals. Int J Diabetes Clin Res 8:140. doi.org/10.23937/23773634/1410140

Accepted: May 08, 2021: Published: May 10, 2021

Copyright: (C) 2021 Ranasinghe U, et al. This is an open-access article distributed under the terms of the Creative Commons Attribution License, which permits unrestricted use, distribution, and reproduction in any medium, provided the original author and source are credited. 
and surgery $[3,4]$.

Hospital-acquired DKA (HADKA) is defined as diabetic ketoacidosis, which occurs during a hospital stay while being admitted and treated for different medical conditions other than DKA [5]. The pathophysiology is related to relative or absolute insulin deficiency and an increase in counter-regulatory hormones. According to the national inpatient audit carried out in England and Wales in 2017, all hospital-acquired DKA should be recorded as a serious incident and undertake root cause analysis [6].

The objectives of this study were to identify the number of HADKA cases in 2 major hospitals in the Hunter New England Health District and conduct a retrospective analysis to identify common contributing factors. We also looked at patient factors, biochemical markers, length of hospital stay and ICU admission rates for the patients with HADKA.

\section{Methods}

The study looked at patients admitted between $1^{\text {st }}$ July 2014 and $30^{\text {th }}$ June 2018 in two major hospitals in the Hunter New England Health District, NSW.

Hospital $\mathbf{A}$ is the principal referral centre and a tertiary hospital for Newcastle and northern New South Wales, Australia. It has several medical and surgical sub-speciality teams, including a dedicated endocrine and specialist diabetes service with an inpatient diabetes educator service. Hospital B is the primary cancer care centre for the Hunter New England Local Health District. It also provides general medicine and general surgical services. It does not have a dedicated diabetes team or diabetes educator service for inpatients.
During the study period, 476,522 patients were admitted, out of which 47,622 had diabetes (9.99\%). The majority of the patients had T2DM ( $n=4,3854,92.1 \%)$.

Hunter New England Health Medical Records Department records all hospital-acquired medical conditions with a condition onset flag. Every patient discharge is evaluated and coded for each active medical issue. The data was cross-examined with the admission notes and discharge letters to confirm if the event did indeed occur. The DKA criteria were $\mathrm{pH} \leq 7.3$, bicarbonate $\leq 18$ $\mathrm{mmol} / \mathrm{L}$ and ketone detection (plasma or urinary ketones).

\section{Inclusion criteria for HADKA:}

1) $\mathrm{pH} \leq 7.3$

2) Bicarbonate $\leq 18 \mathrm{mmol} / \mathrm{L}$

3) Presence of ketones (plasma ketones or urinary ketones)

\section{Exclusion criteria (any of the following):}

1) Presence of DKA on presentation to hospital

2) Acute medical condition to explain acidosis other than DKA (e.g., Acute Kidney Injury)

3) Age $<18$

The factors surrounding the development of HADKA and its complications were obtained by directly reviewing hospital records, patient notes and biochemistry databases. All possible reasons for HADKA were identified and categorised into groups. We adhered to the RCA guidelines from the National Patient Safety Foundation [7]. Once cases were confirmed, we assessed the primary cause (causes) which led to DKA, time

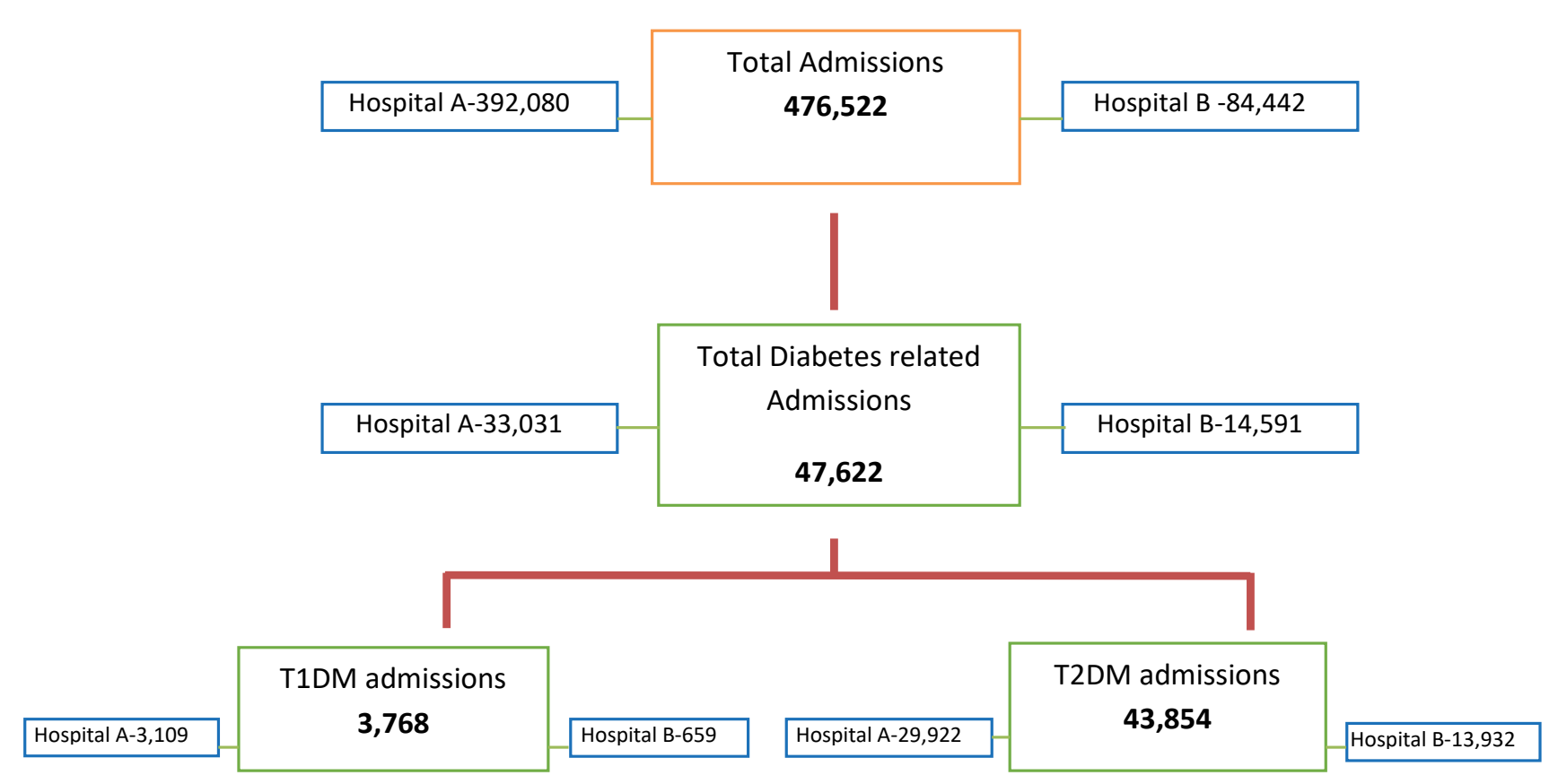

Chart 1: Total Diabetes Admissions. 
to HADKA since the admission (first documented occurrence of acidosis - $\mathrm{pH} \leq 7.3$ ), time taken for resolution, and how healthcare staff investigated and eliminated the causes. We also looked at markers of severity $(\mathrm{pH}$, bicarbonate, glucose), need for intensive care admission and death.

We excluded patients whose primary admission diagnosis was DKA. This included any patient diagnosed in the emergency department or at the time of first post-admission review. We also excluded patients who had comorbidities which significantly contributed to acidosis (e.g., acute kidney injury). We did not exclude patients based on glycaemic range to capture euglycaemic HADKA. We included patients who didn't have documented ketones but satisfied all other DKA inclusion criteria, were treated for DKA and did not have another medical condition responsible for the acidosis. This group of patients was labelled "inferred HADKA".

\section{Statistical Analysis}

Baseline characteristics are presented as means and as absolute and relative percentages. Comparison of biochemical targets, time for recovery and the duration of hospital stay were performed using chi-square test and unpaired T-test. A $P$ value less than 0.05 was considered statistically significant. All the analyses were per- formed with the use of DATAtab (Chart 1).

The study was approved by the Hunter New England Human Research Ethics Committee.

\section{Results}

During the study period, a total of 940 patients were flagged with DKA, out of which 40 had a condition onset flagged for HADKA. Out of these 40 patients, 31 were from Hospital B and only nine from Hospital A (Chart 2).

Out of the 31 patients from Hospital B, only 5 met all the criteria for HADKA (true HADKA). Three did not have a documented ketone (Inferred HADKA); however, we included them as having had HADKA since there was no identifiable alternative medical condition causing acidosis and they were treated for HADKA. Out of the 9 patients from Hospital A, four had true HADKA. The rest ( $n=23$ from Hospital $B$ and $n=5$ from Hospital $A$ ) were wrongly classified as having DKA or had a different acute medical condition other than DKA which was the leading cause of the acidosis.

The overall average age of the group was 64 years (63 years and 66.25 years for Hospital B and Hospital $A$ respectively). The majority of the patients were male $(n=8)$ and only four were female. Only one of the patients was aboriginal. All the patients had a diagnosis of

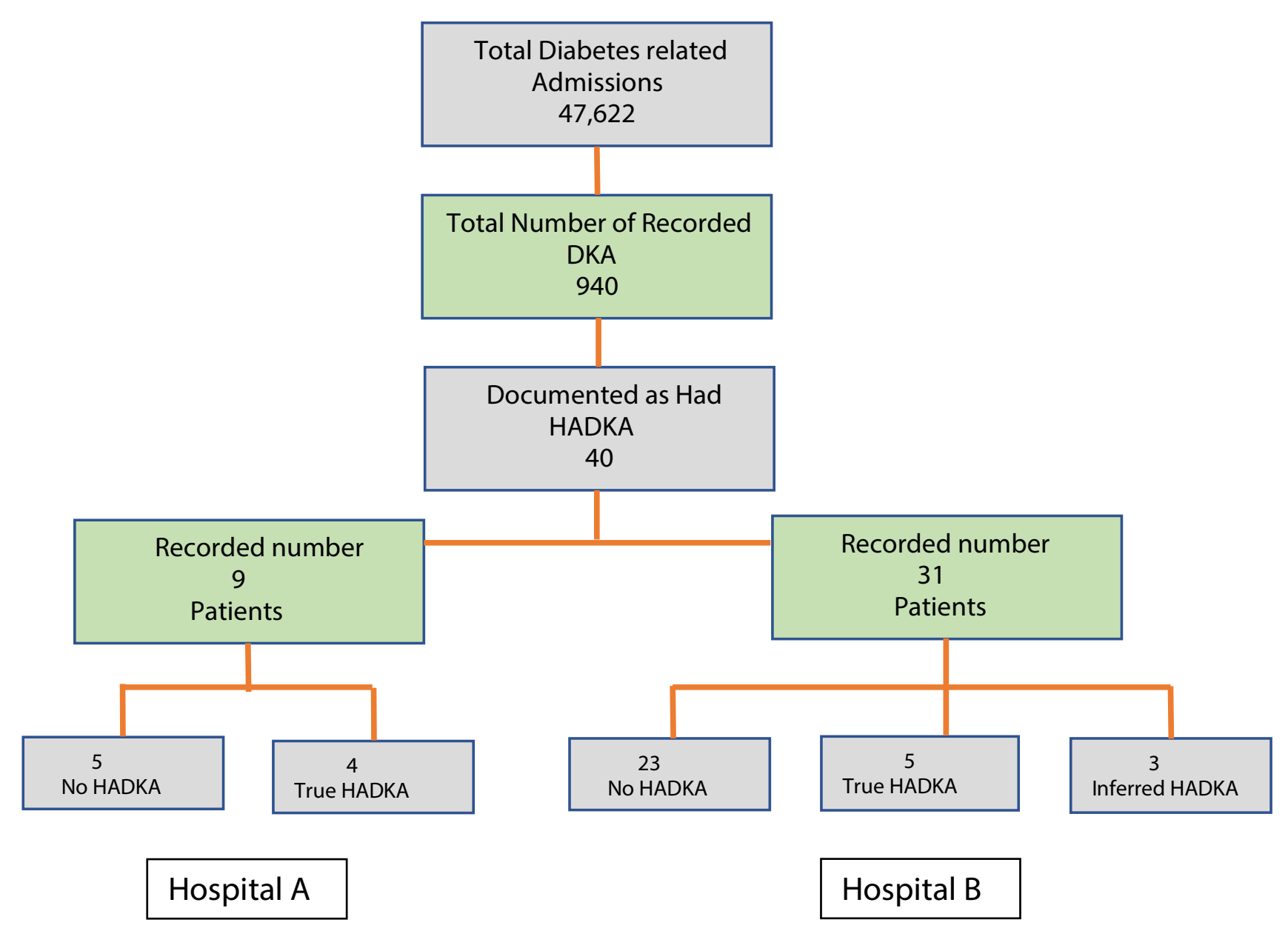

Chart 2: Patient selection. 
diabetes at the time of admission. 9 had T2DM, 2 had T1DM, and 1 had T3CDM (pancreatitis related diabetes). The average $\mathrm{HbA1c}$ (within six months of admission) was $8.42 \%$. The majority of the patients were admitted under a medical speciality $(n=5)$, four under a surgical speciality and three under other specialities (Oncology, Haematology) (Table 1).

Out of the 12 patients, 7 were admitted with an acute medical condition (either infection, acute coronary syndrome, opioid withdrawal, Mallory-Weiss syndrome or pulmonary embolism) and 3 were admitted with an acute surgical disease (acute pancreatitis, abdominal pain, mucositis). Only 2 were elective admissions (coronary artery bypass surgery) while the others were unplanned emergency admissions (Table 2).

We identified associated chronic medical conditions according to the Australian Institute of Health and Welfare which were asthma, back pain, cancer, cardiovascular disease, chronic obstructive pulmonary disease, mental health conditions, osteoporosis and chronic kidney disease. All of the patients had at least one more chronic medical condition other than diabetes. Five of the patients had two additional chronic medical conditions, and another five patients had three additional chronic medical conditions apart from diabetes (Table 3).

Using common cause analysis, the most prevalent reason for DKA was related to an erroneous insulin administration $(n=5,41.66 \%)$. This included withholding insulin $(n=1,8.33 \%)$, insulin dose reduction $(n=3,25 \%)$ and interruption to insulin infusion due to kinking of the access line $(n=1,8.33 \%)$. Another 4 cases were related to infection $(n=4,33.33 \%), 3$ related to SGLT2 inhibitors $(n=3,25 \%), 1$ related to steroid-induced hyperglycaemia ( $n=1,8.33 \%$ ) and 2 cases related to acute stress associated with cardiac disease $(n=2,16.66 \%)$. Two of the patients had more than one contributing factor for developing HADKA (Table 4).

All patients developed HADKA after 24 hours following their admission. The average time taken for the development of HADKA from the time of admission was 3.75 days for Hospital A, and 3.85 days Hospital B.

At the time which the patients had HADKA, the nadir average $\mathrm{pH}$ was 7.19 (need range) and the lowest average bicarbonate was $12.92 \mathrm{mmol} / \mathrm{L}$. The maximum average glucose reading was $17.52 \mathrm{mmol} / \mathrm{L}$ (give range). In the patients who developed HADKA related to SGLT2 inhibitor therapy, the average highest recorded blood

Table 1: Patient demographics.

\begin{tabular}{|c|c|c|c|c|c|}
\hline \multicolumn{2}{|l|}{ Demographics } & Hospital A & Hospital B & All patients & $P$ value \\
\hline \multicolumn{2}{|l|}{ Age } & $66.25(58-74)$ & 63(33-88) & 64.08 & 0.72 \\
\hline \multirow[t]{2}{*}{ Ethnicity } & Aboriginal & 1 & 0 & 1 & \multirow[b]{2}{*}{0.16} \\
\hline & Non-Aboriginal & 3 & 8 & 11 & \\
\hline \multirow[t]{2}{*}{ Sex } & Male & 2 & 6 & 8 & \multirow[b]{2}{*}{0.43} \\
\hline & Female & 2 & 2 & 4 & \\
\hline \multirow[t]{3}{*}{ Diabetes } & T1DM & 1 & 1 & 2 & \multirow{3}{*}{0.45} \\
\hline & T2DM & 3 & 6 & 9 & \\
\hline & Other & & 1 & 1 & \\
\hline \multicolumn{2}{|l|}{ MeanHbA1c } & $7.42 \% \pm 2.26$ & $9.22 \% \pm 2.13$ & $8.42 \% \pm 2.37$ & 0.31 \\
\hline \multirow[t]{3}{*}{ Admission Team } & Medical & 1 & 4 & 5 & \multirow{3}{*}{0.81} \\
\hline & Surgical & 3 & 1 & 4 & \\
\hline & Other & 0 & 3 & 3 & \\
\hline
\end{tabular}

Table 2: Type of hospital admission.

\begin{tabular}{|l|l|l|l|l|}
\hline & Hospital A & Hospital B & All patients & P value \\
\hline Acute medical admission & 2 & 5 & 7 & 0.711 \\
\hline Acute surgical admission & & 3 & 3 & \\
\hline Elective procedure & 2 & & 2 & \\
\hline
\end{tabular}

Table 3: Associated chronic medical conditions.

\begin{tabular}{|l|l|l|l|}
\hline Number of chronic medical conditions (excluding diabetes) & Hospital A & Hospital B & All patients \\
\hline 0 & & & \\
\hline 1 & 1 & 1 & 2 \\
\hline 2 & 3 & 2 & 5 \\
\hline 3 & & 5 & 5 \\
\hline
\end{tabular}


Table 4: Factors contributing to HADKA.

\begin{tabular}{|l|l|l|l|l|}
\hline Cause & Hospital A & Hospital B & All patients \\
\hline \multirow{2}{*}{ Insulin administration } & Withholding insulin & 0 & 1 & 1 \\
\cline { 2 - 5 } & Insulin dose reduction & 0 & $2,(3)$ & $2,(3)$ \\
\cline { 2 - 5 } & Interruption to insulin infusion & 1 & 0 & 1 \\
\hline Infection & & $3,(4)$ & $3,(4)$ \\
\hline Steroid use & $2,(3)$ & 1 & 1 \\
\hline SGLT2 inhibitor & $(1)$ & 0 & $2,(3)$ \\
\hline Acute stress from disease & & 1 & $1,(2)$ \\
\hline
\end{tabular}

(In brackets - when more than one causative factor is present).

Table 5: Biochemical and clinical outcomes of HADKA.

\begin{tabular}{|c|c|c|c|c|}
\hline & Hospital A & Hospital B & All patients & $P$ value \\
\hline $\mathrm{pH}$ & $7.24 \pm 0.05$ & $7.13 \pm 0.06$ & $7.19 \pm 0.07$ & 0.089 \\
\hline Glucose & $16.12 \pm 10.63$ & $18.22 \pm 3.84$ & $17.52 \pm 6.43$ & 0.6 \\
\hline Bicarbonate & $15.42 \pm 2.43$ & $11.67 \pm 6.38$ & $12.92 \pm 5.56$ & 0.29 \\
\hline Time to recovery & 3.5 days \pm 1.73 & 3.25 days \pm 2.86 & 3.33 days \pm 2.46 & 0.87 \\
\hline $\begin{array}{l}\text { Time to DKA from } \\
\text { admission }\end{array}$ & 3.75 days \pm 2.88 & 3.85 days \pm 2.50 & 3.83 days \pm 2.50 & 0.94 \\
\hline Duration of admission & 15.25 & 15.83 & 15.6 & 0.84 \\
\hline ICU admission & 3 & 7 & 10 & 0.69 \\
\hline Mortality & 0 & 2 & 2 & 0.31 \\
\hline
\end{tabular}

Plus-minus values are means \pm SD.

Table 6: Specialized diabetes care for patients with HADKA.

\begin{tabular}{|l|l|l|l|}
\hline & $\begin{array}{l}\text { Hospital } \\
\text { A }\end{array}$ & $\begin{array}{l}\text { Hospital } \\
\text { B }\end{array}$ & $\begin{array}{l}\text { All } \\
\text { patients }\end{array}$ \\
\hline $\begin{array}{l}\text { Diabetes educator } \\
\text { review }\end{array}$ & 4 & 0 & 4 \\
\hline $\begin{array}{l}\text { Inpatient diabetes team } \\
\text { review }\end{array}$ & 4 & 0 & 4 \\
\hline
\end{tabular}

glucose reading was $10.8 \mathrm{mmol} / \mathrm{L}$. 10 out of 12 patients needed ICU admission during their management of HADKA. 2 patients died after developing HADKA. The average time for HADKA resolution was 3.33 days. The average duration of hospital admission was 15.6 days (Table 5).

Out of the total of 12 patients, 11 were treated with insulin infusion and 1 was treated with just subcutaneous insulin. All the patients from Hospital A were reviewed by the diabetes educator and the hospital diabetes team. In Hospital B, however, none of the patients were reviewed by a diabetes educator or a team specialised in diabetes care (Table 6).

\section{Discussion}

Hospital-acquired DKA is a dangerous complication which may present itself in diabetic patients who are admitted to a hospital. Diabetes is a major comorbidity in patients who get admitted to a hospital. In our study, 9.99\% of total hospital admissions had diabetes, similar to other major studies (10\%) [8]. According to the National Diabetes Inpatient Audit England and Wales 2017, DKA during the hospital stay was around $4 \%$ [6]. In our study, the overall HADKA rate was low $(0.02 \%)$, which might be due to underdiagnosis and poor electronic data recording rather than a true reflection of the prevalence of HADKA. However, Hospital B had many more occurrences than Hospital A which has a dedicated diabetes unit. In Hospital A, the HADKA occurrence was $0.01 \%$ compared to $0.05 \%$ in Hospital B ( $p$-value .007).

Insulin errors resulted in most cases of HADKA. Insulin is vital for patients with T1DM and some T2DM patients, but administration errors can lead to severe complications. An Australian survey including 11 hospitals and 2,308 adult inpatients in metropolitan Melbourne reported $25 \%$ (range: $15.7-35.1 \%$ ) of diabetes prevalence, and almost half of them were treated with insulin alone or in combination with oral hypoglycaemic agents [9]. There is clear evidence to suggest that insulin errors are the most common medication error in hospitals. As per the National Patient Safety Agency in the United Kingdom, a total of 16,600 incidents involving insulin were identified in England and Wales from November 2003 to November 2009, amongst which 18 incidents had fatal and devastating outcomes and 1,042 incurred moderate harm [10]. The National Diabetes Inpatient Audit in 2017 revealed that $22.7 \%$ of inpatient medication charts had at least one insulin medication 
error [6]. The incidence was even higher in a study done at St Vincent's Hospital in Sydney, Australia which showed that $41.5 \%$ of insulin charts revealed one or more prescription or administration errors in 2013 [11].

The most common cause of insulin administration errors found in our study was inappropriate dose reduction. Glucose metabolism is maintained by an interaction of glucoregulatory hormones - insulin and counterregulatory hormones (glucagon, cortisol, growth hormone and catecholamines). Insulin controls hepatic glucose production by suppressing hepatic gluconeogenesis and glycogenolysis. In insulin-sensitive tissues such as muscle, insulin promotes protein anabolism, glucose uptake and glycogen synthesis while inhibiting glycogenolysis and protein breakdown. Insulin is a potent inhibitor of lipolysis, free fatty acid oxidation and ketogenesis. Counterregulatory hormones (glucagon, cortisol, growth hormone and catecholamines) also play an essential role in regulating glucose production and utilisation. Glucagon is the most important glycogenolytic hormone; therefore, it regulates hepatic glucose production during normal state and in every state of hyperglycaemia. During stress, an excess concentration of counter regulatory hormones results in altered carbohydrate metabolism by inducing insulin resistance, increasing hepatic glucose production, downregulation of insulin release from beta-cells and reduction of peripheral glucose utilisation $[12,13]$. Patients are under constant stress during their hospital stay, which results in the release of an excess concentration of counterregulatory hormones leading to hyperglycaemia. Pre-admission insulin doses are insufficient to maintain the plasma glucose in the target range, and dose escalation should happen promptly to avoid hyperglycemic com- plications such as HADKA (Figure 1).

One of the most remarkable findings was that most of the patients who developed HADKA were elderly patients with T2DM. It is mostly believed that DKA is a serious complication of young T1DM patients rather than of patients with T2DM. DKA occurs when there is a relative deficiency of insulin and imbalance between insulin and counter-regulatory hormones. Older adults with T2DM can have low intrinsic insulin production from reduced beta-cell mass and are vulnerable in developing DKA during stressful situations such as hospital admissions. In a study which looked at 138 patients admitted for moderate-to-severe DKA in a major university hospital in the United States, 30 patients had type 2 diabetes and infections were present in $48.4 \%$ of the type 2 diabetes admissions [14]. Lack of awareness among the medical staff that DKA can occur with T2DM may be responsible for these patients' insulin errors and this will likely result in delayed diagnosis as a consequence of failure to order tests on ketone levels.

Out of the 8 HADKA cases from hospital B, 3 did not have recorded ketones even when the BGLs were over $20 \mathrm{mmol} / \mathrm{L}$ with acidosis. This is likely to result in underdiagnosis of HADKA, especially in milder forms. This could be related to traditional teaching that DKA is only seen in type 1 diabetes and not in type 2 diabetes. Studies show that DKA in the elderly is often associated with sepsis, atrial fibrillation, polypharmacy, acute kidney injury (AKI), and dementia [15]. As a result, DKA in elderly patients is often masked by various clinical conundrums, which may mask the disease pathology and lead to a delay in the diagnosis and management of DKA.

\section{Pathophysiology of Hospital-acquired Diabetic ketoacidosis}

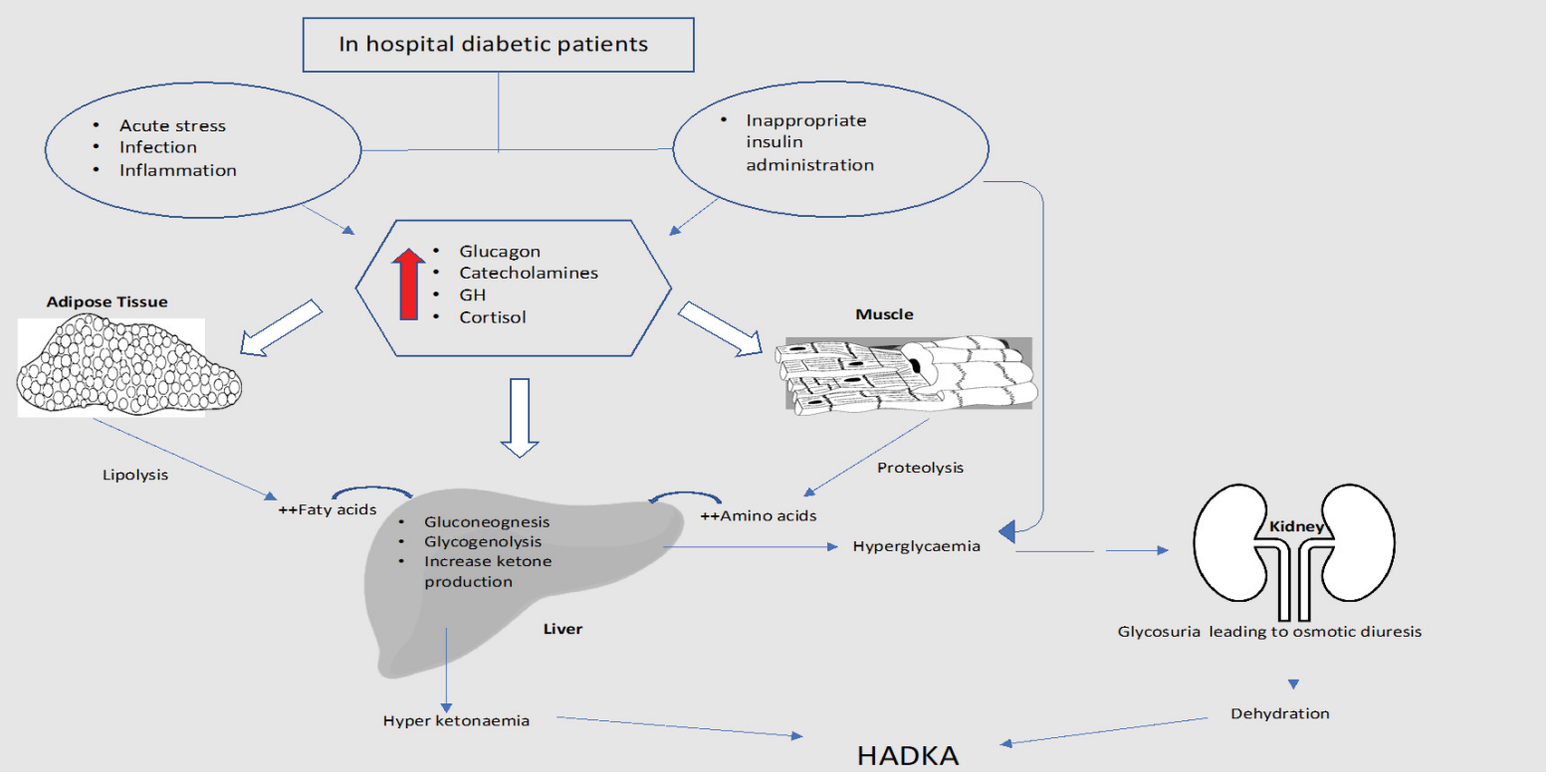

Figure 1: Pathophysiology of Hospital-acquired Diabetic ketoacidosis. 
The NSW insulin chart guidelines recommend checking ketones if T1DM with BGLs is higher than 15 $\mathrm{mmol} / \mathrm{L}$. It will be necessary to review similar studies in the future and revisit guidelines to recommend checking ketones for all patients with BGLs above $15 \mathrm{mmol} / \mathrm{l}$ in the hospital.

There were 3 HADKA cases related to SGLT2 inhibitor therapy. All 3 patients developed euglycemic DKA (average highest $B G L=10.8 \mathrm{mmol} / \mathrm{L}$ ), a known severe complication of this medication [16]. According to the European Medicines Agency (EMA) announcement, as of May 2015, a total of 101 cases of DKA have been reported worldwide in T2DM patients treated with SGLT2 inhibitors, with an estimated exposure over 0.5 million patient-years [17]. With new evidence on the benefits of using SGLT2 inhibitors in both diabetic and non-diabetic patients (heart failure), the prevalence of HADKA is- likely to increase [18]. All medical teams should be aware of the DKA risk associated with SGLT2 inhibitors, and the medication should be discontinued at the time of admission in high-risk patients. In addition to this, hospital insulin charts and guidelines should highlight the risks associated with SGLT2 inhibitors.

Hospital A had a lower number of HADKA cases, faster recovery from DKA as well as less number of deaths after HADKA compared to Hospital B. Patients from Hospital B were sicker (worse $\mathrm{pH}$, bicarbonate and blood glucose) and needed ICU management. All patients with HADKA from Hospital A were reviewed by the inpatient diabetes team and the diabetes educator. On the contrary, none of the Hospital B patients had any input from a specialised diabetes service or a diabetes educator. A dedicated diabetic team and a diabetes educator likely helped lower cases of HADKA and promote faster recovery. A study from 2018 showed that an inpatient specialised management team reduced secondary diabetes complications as well as inpatient costs, and also improved care transition and adherence to follow-up [19]. According to Levetan, et al. obtaining an endocrinology consultation, either alone or as part of a multidisciplinary diabetes team (endocrinologist, diabetes nurse educator, and a registered dietitian), reduced the length of hospital stay in patients admitted with the principal diagnosis being diabetes or its complications (hyperosmolar state, diabetic ketoacidosis and uncontrolled diabetes) [20]. In the same study, the average length of stay of the diabetes team patients was $3.6 \pm$ 1.7 days compared to $8.2 \pm 6.2$ days for patients in the no-consultation group and $5.5 \pm 3.4$ days for the patients who received a traditional individual endocrine consultation. The reasons outlined as contributing towards a shortened length of stay were more rapid normalisation of glucose levels, more efficient transition from intravenous to subcutaneous insulin, a faster transition to definitive insulin or an oral medication regimen and more effective diabetes survival skills. In a study looking at more than 100,000 hospitalised patients with diabe- tic ketoacidosis, patients cared for by endocrinologists had a shorter hospital stay and a lower readmission rate for DKA than the patients managed by generalists [21]. These studies also highlight that there is a substantial potential opportunity for cost savings from improved hospital outcomes, reduced mortality and shortened length of stay for patients with diabetes and hospital-related hyperglycaemia.

Given the variety of clinical situations in the inpatient setting, it is common for medical centres to establish an inpatient diabetes team dedicated to managing inpatient hyperglycaemia through these transitions. There is ample evidence that these teams improve inpatient glucose ranges and with early consultation, they may reduce lengths of hospital stay [22]. Perhaps most significantly, using hospitalisation as an opportunity to educate a patient about diabetes and to optimise their treatment may improve long-term outpatient glycaemic control as well.

According to the Australian Institute of Health and Welfare, between 2014 and 2015, the average length of hospital stay in a public hospital was 2.7 days [2]. The average length of stay for patients with diabetes was 2.5 to 3 days longer than for those without, irrespective of age, socioeconomic status, admission type or case complexity [8]. In our study, the average length of hospital stay was 15.6 days which is much higher than the length of stay for the general admission patients. All of the patients had at least one other chronic medical condition and more than $80 \%$ of the patients had two or more chronic medical statuses apart from diabetes, at the time of admission. This is unlikely with young T1DM patients who mostly do not have chronic medical conditions. The average length of stay in the hospital for DKA among children and young people with type 1 diabetes was 2.9 days, with the length of stay similar for males and females (2.8 and 2.9 days, respectively) [2]. A retrospective study looking at trends in hospital admission for diabetic ketoacidosis (DKA) in adults with type 1 diabetes mellitus (T1DM) and type 2 diabetes mellitus (T2DM) from 1998 to 2013 in England showed that adults with T1DM were more likely to be discharged earlier than adults with T2DM [23].

The majority of patients (83.3\%) in our study needed ICU admission. It is estimated that about $2 \%$ to $20 \%$ of acute hospital admissions receive ICU care during their hospital stay [24]. A study looking at the New York State Inpatient Database between 2005 and 2007 identified that a median of $11.8 \%$ of all inpatient admissions needed ICU management during their hospitalisation [25]. The study also pointed out that $52.6 \%$ of patients with DKA required ICU care. The majority of the patients were young adults. Patients with HADKA may be likely to have higher ICU admission rates than patients admitted with DKA due to older age of presentation, a greater number of comorbidities and delay in identifying and 
escalating treatment. Future studies using randomised prospective design are needed to verify these results.

There were several limitations to this study. With regards to recruitment, the sample number is small, making it difficult to draw firm conclusions. This study also leads us to believe that there have been several false negatives that were not recruited. This is because a considerable number of patients were wrongly coded with HADKA. Although this represents a false positive and was therefore excluded, the process of coding patients with HADKA had errors. This process is first based on coders reviewing discharge summaries for mention of DKA. Subsequently, these patients are cross-correlated with whether they also had a health staff-initiated flagging of 'hospital-related event'. HADKA was then flagged if both occurred for the same patient. This system is open to error. Another limitation of this study is the variation in the documentation of ketone levels for these patients. Of the three inclusion criteria for HADKA, only ketone levels are not automatically stored electronically in the laboratory database. Some patients had only urinary ketone measurements. Documentation of this finding was variable. This could have resulted in the underdiagnosis of HADKA. An attempt to minimise this effect was made by having a group of 'inferred HADKA' patients.

\section{Future Directions}

HADKA remains a serious underdiagnosed nosocomial complication with high morbidity and mortality. All hospitals should identify cases of HADKA and conduct root cause analysis and common cause analysis to understand all contributory factors. Better electronic documentation and coding to identify HADKA will ease this process. The National Safety and Quality Health Service (NSQHS) classifies a hospital-acquired complication (HAC) as an incident for which clinical risk mitigation strategies may reduce (but not necessarily eliminate) the risk of that complication occurring. It recognises 16 lists of high-priority HACs which were created through a clinician-driven process. Under the sub-category of endocrine, it recognises malnutrition and hypoglycemia as high priority HACs [26]. HADKA should be recognised as well.

All healthcare practitioners should be aware of the risk of HADKA in diabetic patients, and all cases of HADKA should be reported as significant incidents and alongside being reported in the Incident Information Management System. Online e-learning modules discussing HADKA can help increase vigilance among healthcare workers, in preventing HADKA. Inpatient hyperglycaemia guidelines and insulin prescribing charts should highlight the necessity to monitor ketones for all patients when the BGLs are above $15 \mathrm{mmol} / \mathrm{L}$ and there is a risk of euglycaemic diabetic ketoacidosis with SGLT2 inhibitors. Establishing a dedicated inpatient diabetes/endocrine service will help prevent serious diabetic complications such as HADKA. A good start to recognising the true HADKA burden in our hospitals is designing a prospective study to capture and analyse this problem.

\section{Summary}

HADKA remains a serious hospital-acquired complication with high morbidity and mortality. The National Diabetic Audit by NHS, UK strongly recommends recording all hospital-acquired DKA as Serious Incidents and undertake root cause analysis [6]. Elderly patients with comorbidities have the greatest risk of developing HADKA with poorer outcomes. Even though HADKA remains a serious complication, there is a lack of studies looking at root cause analysis in Australia. While policy interventions may be necessary to prevent HADKA, our results point to the need for future work to clarify our findings and identify practical solutions.

\section{References}

1. Australian Institute of Health and Welfare (2020) Diabetes.

2. Australian Institute of Health and Welfare (2016) Diabetic ketoacidosis (DKA) among children and young people with type 1 diabetes.

3. Umpierrez GE, Isaacs SD, Bazargan N, You X, Thaler LM, et al. (2002) Hyperglycemia: An independent marker of in-hospital mortality in patients with undiagnosed diabetes. J Clin Endocrinol Metab 87: 978-982.

4. Yamada T, Shojima N, Noma H, Yamauchi T, Kadowaki T (2017) Glycemic control, mortality, and hypoglycemia in critically ill patients: A systematic review and network meta-analysis of randomized controlled trials. Intensive Care Med 43: 1-15.

5. Zimmerman C (2019) latrogenic complications of diabetes mellitus: An examination of hospital-acquired diabetic ketoacidosis and severe outpatient hypoglycemia. Yale Medicine Thesis Digital Library, 3546.

6. Digital NHS (2018) National Diabetes Inpatient Audit 2017 Report. Diabetes, UK.

7. National Patient Safety Foundation (2015) RCA2: Improving root cause analysis and actions to prevent harm.

8. Comino EJ, Harris MF, Islam F, Tran DT, Jalaludin B, et al. (2015) Impact of diabetes on hospital admission and length of stay among a general population aged 45 year or more: A record linkage study. BMC Health Serv Res 15: 12.

9. Bach LA, Ekinci El, Engler D, Gilfillan C, Hamblin PS, et al. (2014) The high burden of inpatient diabetes mellitus: The Melbourne Public Hospitals Diabetes Inpatient Audit. Med J Aust 201: 334-338.

10. Cousins D, Rosario C, Scarpello J (2011) Insulin, hospitals and harm: A review of patient safety incidents reported to the National Patient Safety Agency. Clin Med (Lond) 11: 28-30.

11. Taylor JE, Campbell LV, Zhang L, Greenfield JR (2018) High diabetes prevalence and insulin medication errors in hospital patients. Intern Med J 48: 1529-1532.

12. Kitabchi AE, Umpierrez GE, Miles JM, Fisher JN (2009) Hyperglycemic crises in adult patients with diabetes. Diabetes Care 32: 1335-1343.

13. Dorin RI, Qualls CR, Torpy DJ, Schrader RM, Urban FK (2015) Reversible increase in maximal cortisol secretion rate in septic shock. Crit Care Med 43: 549-556. 
14. Newton CA, Raskin P (2004) Diabetic ketoacidosis in type 1 and type 2 diabetes mellitus: Clinical and biochemical differences. Arch Intern Med 164: 1925-1931.

15. Sehgal V, Ulmer B (2019) Clinical conundrums in the management of diabetic ketoacidosis in the elderly. J Transl Int Med 7: 10-14.

16. Gajjar K, Luthra P (2019) Euglycemic diabetic ketoacidosis in the setting of SGLT2 inhibitor use and hypertriglyceridemia: A case report and review of literature. Cureus 11 : e4384.

17. Rosenstock J, Ferrannini E (2015) Euglycemic diabetic ketoacidosis: A predictable, detectable, and preventable safety concern with SGLT2 inhibitors. Diabetes Care 38: 1638-1642.

18. Zannad F, Ferreira JP, Pocock SJ, Anker SD, Butler J, et al. (2020) SGLT2 inhibitors in patients with heart failure with reduced ejection fraction: A meta-analysis of the EMPEROR-Reduced and DAPA-HF trials. Lancet 396: 819-829.

19. Bansal V, Mottalib A, Pawar TK, Abbasakoor N, Chuang E, et al. (2018) Inpatient diabetes management by specialized diabetes team versus primary service team in non-critical care units: Impact on 30-day readmission rate and hospital cost. BMJ Open Diabetes Res Care 6: e000460.
20. Levetan CS, Salas JR, Wilets IF, Zumoff B (1995) Impact of endocrine and diabetes team consultation on hospital length of stay for patients with diabetes. Am J Med 99: 22-28.

21. Levetan CS, Passaro MD, Jablonski KA, Ratner RE (1999) Effect of physician specialty on outcomes in diabetic ketoacidosis. Diabetes Care 22: 1790-1795.

22. Koproski J, Pretto Z, Poretsky L (1997) Effects of an intervention by a diabetes team in hospitalized patients with diabetes. Diabetes Care 20: 1553-1555.

23. Zhong VW, Juhaeri J, Mayer-Davis EJ (2018) Trends in hospital admission for diabetic ketoacidosis in adults with type 1 and type 2 diabetes in England, 1998-2013: A retrospective cohort study. Diabetes Care 41: 1870-1877.

24. Wunsch $\mathrm{H}$, Linde-Zwirble WT, Harrison DA, Barnato AE, Rowan KM, et al. (2009) Use of intensive care services during terminal hospitalizations in England and the United States. Am J Respir Crit Care Med 180: 875-880.

25. Gershengorn HB, Iwashyna TJ, Cooke CR, Scales DC, Kahn JM, et al. (2012) Variation in use of intensive care for adults with diabetic ketoacidosis*. Crit Care Med 40: 20092015.

26. Australian Commission on Safety and Quality in Health Care (2019) Hospital-Acquired Complications. 